\title{
Une avancée optique pour la mesure de la salinifé absolue des océans
}

\author{
Marc LE MENN - SHOM (Service hydrographique et océanographique de la Marine) \\ Jean-Louis de BOUGRENET de la TOCNAYE - Département Optique de Télécom Bretagne \\ Philippe GROSSO - Département Optique de Télécom Bretagne - UMR CNRS 6082 FOTON \\ Laurent DELAUNEY et Christian PODEUR - IFREMER \\ Patrice BRAULT et Olivier GUILLERME - NKE
}

La salinité est un paramètre clé pour déterminer les propriétés physiques de l'eau de mer. Sa connaissance permet notamment d'étudier la circulation des grands courants marins. Les transports de chaleur et de masse qui leur sont associés sont régis principalement par leurs variations de salinité ou de masse volumique, les deux paramètres étant liés. L'importance que revêt de nos jours l'étude des variations climatiques et des variations de salinité liées par exemple à la fonte de la calotte glacière ainsi que les biais constatés sur les mesures de salinité, ont conduit les océanographes à s'intéresser au développement de nouveaux capteurs fiables, précis et utilisables in situ, pour surveiller l'évolution des phénomènes océaniques.

\section{Salinité pratique et salinité absolve}

En se basant sur les travaux de l'International Association for the Properties of Water and Steams, le calcul des propriétés de l'eau de mer a été redéfini [1] par un groupe de travail de l'UNESCO baptisé SCOR/IAPSO Working Group 127 (WG 127). Ils ont créé le TEOS-10 ou International Thermodynamic Equation Of Seawater, basé sur le potentiel de Gibbs, fonction décrivant l'état thermodynamique d'un système. Pour l'eau de mer, cette fonction dépend de la salinité absolue, de la température et de la pression. Jusqu' à présent, la salinité des océans est calculée par les formules de l'échelle pratique de salinité de 1978 (PSS78) basée sur des mesures du rapport de conductivité de l'échantillon sur celle d'une eau standard. Les valeurs de salinité pratique (quantité sans dimension) sont utilisées pour évaluer les propriétés thermodynamiques du modèle océanique.

Elles présentent cependant plusieurs inconvénients. D'une part, elles ne prennent pas en compte les composés non ioniques dissous dans l'eau de mer, ce qui induit des écarts entre la notion de salinité absolve (exprimée en $\mathrm{g} / \mathrm{kg}$ ) et celle de salinité pratique. D'autre part, la conduc- tivité dépendant fortement de la température, il est très difficile d'aligner les temps de réponse des capteurs de température et de conductivité, ce qui crée des artéfacts sur les valeurs de salinité calculées avec la PSS78, surtout quand les mesures sont effectuées dans des thermoclines bien marquées. Même quand ces valeurs sont corrigées par des algorithmes constructeurs, les erreurs subsistant sur la salinité pratique peuvent atteindre en moyenne 0,017 , après correction, dans des zones de forts gradients thermiques. Enfin, il a été démontré que l'incertitude de 0,002 affichée sur la salinité des bouteilles d'eau standard ne pouvait être garantie que sur une courte période et que de plus, la conductivité de l'eau standard ne peut être rattachée qu'avec une incertitude élargie de 0,021 (rapportée

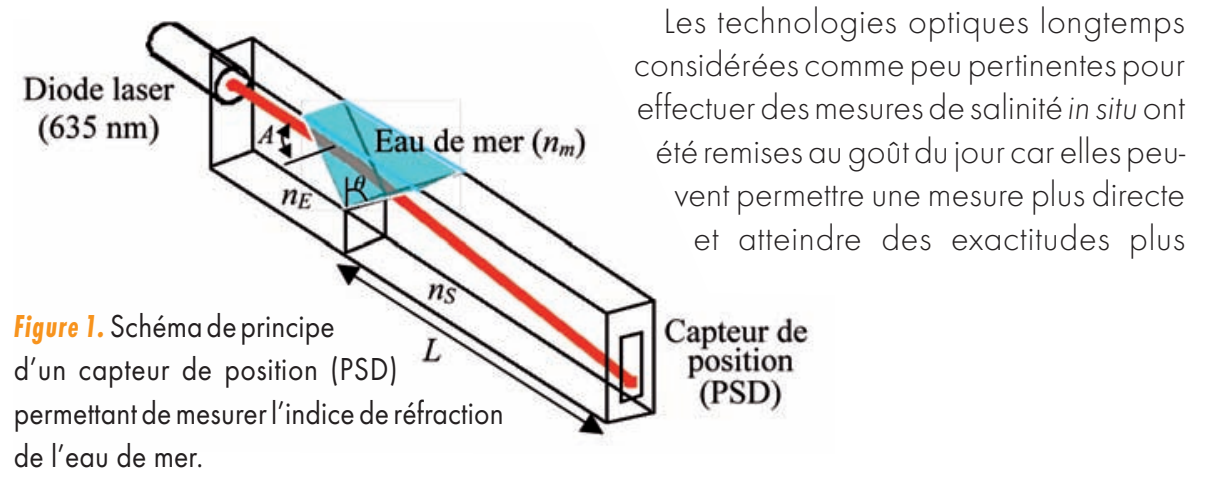

en valeur de salinité pratique), par rapport aux références internationales de conductivité. Ce dernier élément fait qu'à l'avenir, il estfort probable qu'une valeur de masse volumique sera inscrite sur les bouteilles d'eau standard, et que cette grandeur sera un moyen de rattachement de la salinité au SI.

\section{Retour à une solution optique : la réfractométrie haute résolution}

Masse volumique et salinité absolve de I'eau de mer ne sont pas directement mesurables in situ, mais l'indice de réfraction, lié d'une part à la masse volumique et d'autre part à la salinité absolve, peut l'être, et c'est une variable 5 à 10 fois moins sensible aux variations de température que la conductivité.

Les technologies optiques longtemps considérées comme peu pertinentes pour effectuer des mesures de salinité in situ ont té remises au goût du jour car elles peuent permettre une mesure plus directe 
grandes. Différentes méthodes existent pour mesurer in situ l'indice de réfraction - réseaux de Bragg sur fibre, analyse du gain Brillouin - toutes conduisant toutefois à des incertitudes de mesure insuffisantes $\left(5.10^{-5}\right)$. Des résolutions importantes (quelques $10^{-7}$ ) sont possibles par interférométrie mais sur des gammes trop faibles d'indice. L'apparition récente de capteurs de position (PSD) a permis d'envisager une réfractométrie avec un équivalent de 40000 points de résolution. De tels composants permettent des exactitudes de positionnement du faisceau incident (par exemple celui d'une diode laser) variant entre 0,1 et 0,3 $\mu \mathrm{m}$. Ainsi, si le faisceau laser parcourt, après réfraction dans l'eau de mer, tout le PSD, pour une salinité comprise entre 0 et $40 \mathrm{~g} / \mathrm{kg}$, une résolution absolve sur la valeur de la salinité absolve de l'ordre du mg/kg lou de 0,5 ppm pour l'indice de réfraction) peut être atteinte, ce qui répond aux exigences du WG 127 [2] à savoir : incertitude sur l'indice de réfraction 1 ppm à pression atmosphérique et 3 ppm à haute pression. II suffit donc de dimensionner un réfractomètre (figure 1) pour que, dans la gamme de salinité requise, le faisceau laser parcoure entièrement le PSD.

La variation angulaire du faisceau laser après double réfaction ( $d r$ ) et la variation d'indice de réfraction de l'eau de mer $d n_{m}$ sont liées par les relations de SnellDescartes suivantes:

$n_{E} \sin (A)=n_{m} \sin (i)$ et $\sin (r)=\frac{n_{m}}{n_{S}} \sin (2 \theta-i)$

$n_{E}$ et $n s$ sont les indices du prisme d'entrée et de sortie. Un réfractomètre à double prisme permet, en choisissant des verres de coefficients thermo-optiques identiques mais de signe opposé, de rendre la mesure insensible à la température.

À partir des relations de Snell-Descartes, on peut montrer que la variation de l'indice de réfraction de l'échantillon d'eau de mer est liée à la variation de position sur le PSD.

Cette relation tient compte de tous les paramètres opto-géométriques $\left(L, A, n_{E}, n_{S}\right.$, $n_{m}$ et $\theta$ ) auxquels il faut ajouter la longueur d'onde laser. La plupart de ces paramètres dépendent des paramètres environnementaux que sont la température et la pression. Théoriquement, la longueur $L$ peut être ajustée pour une résolution requise, mais le paramètre principal qui agit sur la résolution de l'indice de réfraction reste l'angle d'incidence $A$. Un bon compromis consiste à choisir $A$ entre le minimum de déviation et la réflexion totale. Le minimum de déviation requiert de longs prismes pour couvrir le PSD et la réflexion totale risque de déformer le faisceau, à la jonction des prismes. La modélisation montre que pour un angle de $50^{\circ}$, la variation de l'indice de réfraction de l'échantillon d'eau de mer est de l'ordre du ppm, c'est-à-dire la précision requise par le WG 127.

Le dernier paramètre à considérer est la longueur d'onde de la diode laser. Celleci augmente de $0,2 \mathrm{~nm} /{ }^{\circ} \mathrm{C}$ ce qui diminue l'indice de réfraction des verres. Connaissant la température du laser, cette dérive peut être corrigée électroniquement. Pour une température et une longueur d'onde données, les indices de réfraction ainsi calculés permettent d'obtenir la variation d'indice de réfraction de l'échantillon. Les capteurs optiques, comme on le voit, fournissent un accès direct à la salinité absolue contrairement aux capteurs de conductivité qui présentent des erreurs intrinsèques d'environ $0,16 \mathrm{~g} / \mathrm{kg}$ sur la salinité absolve dans certaines régions océaniques et exigent des incertitudes sur la température et la pression respectivement de $2.10^{-3}{ }^{\circ} \mathrm{C}$ et 2 dbar pour calculer la salinité pratique.
En comparaison, le réfractomètre a la même exigence sur l'incertitude en pression mais se contente pour la température d'une incertitude de $2.10^{-2}{ }^{\circ} \mathrm{C}$.

En tenant compte de tous ces paramètres nous avons conçu un réfractomètre à double prisme dont le schéma est présenté en figure 2

Deux miroirs réalisés par dépôt d'or permettent de placer diode laser et PSD du même côté, facilitant la réalisation d'un instrument compact et immergeable.

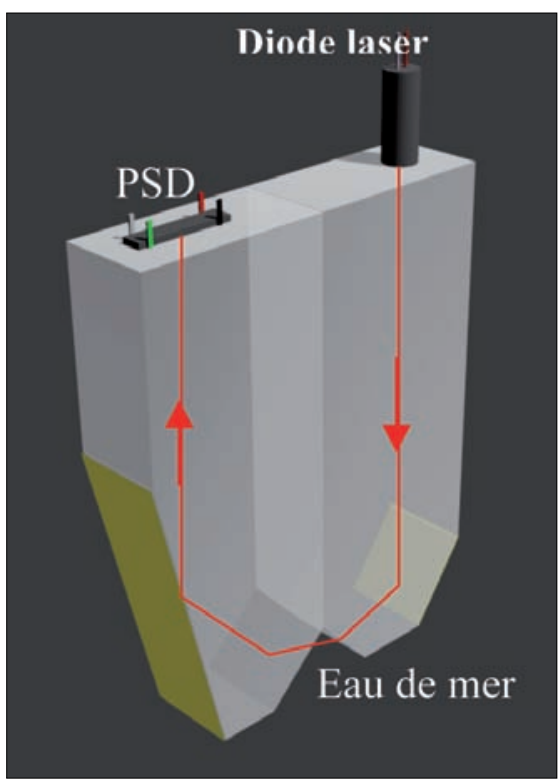

Figure 2. Schéma du réfractomètre à double prisme.

\section{Encadré Projet NOSS (NKE Optical Salinity Sensor)}

NOSS est né d'une collaboration entre le SHOM et Télécom Bretagne. Porté par la Société NKE, qui assure le développement des prototypes, le projet a été labéllisé par le Pôle-Mer Bretagne et financé par le Ministère de l'Industrie (DGE). L'Ifremer a rejoint le consortium pour la partie essais aux conditions d'environnement et intégration mécanique. Le bilan académique compte 2 brevets, 5 publications (ACL), 4 communications et 2 thèses.

Capteur NOSS intégré (photo de gauche) et monté sur profileur PROVOR (photo de droite).

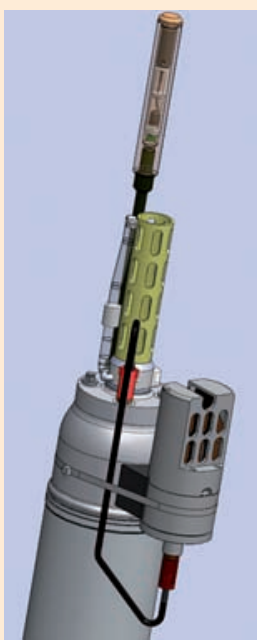




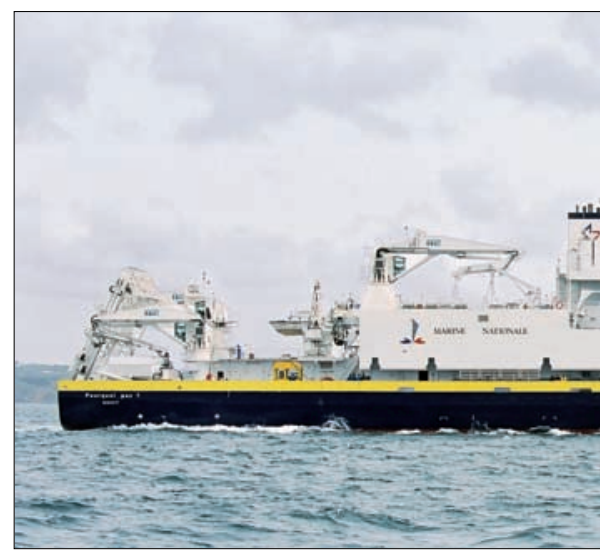

Figure 3. Des essais du prototype NOSS ont été réalisés au printemps 2010. En haut, le Pourquoi Pas, navire océanographique de l'lfremer et de la Marine Nationale. À droite, le prototype NOSS monté sur une rosette.

Ce capteur, baptisé NOSS /voir encadrél, né d'une collaboration entre le SHOM (Service hydrographique et océanographique de la Marine) et Télécom Bretagne, a été intégré par l'Ifremer pour être utilisable in situ, sur différents porteurs : bouées de surface, observatoires sous-marins, profileurs Provor (flotteur dérivant équipé de capteurs de température, conductivité et pression qui envoie ses données, quand il revient en surface périodiquement, par des liaisons satellites). Compacité, résistance à la pression et à la corrosion, inertie thermique ont été des préoccupations majeures lors de la conception. Le capteur a été testé sous pression à l'Ifremer jusqu'à 350 bar. Un circuit électronique spécifique conçu par la Société NKE, permettant des mesures à haute fréquence $(24 \mathrm{~Hz})$, I'a rendu compatible avec un profileur CTD (conductivity, temperature, depth). Un soin particulier a été apporté à l'électronique, pour réduire la consommation d'énergie et le bruit de mesure. Le capteur a été testé en laboratoire pour évaluer les effets indésirables dus à la fluorescence et à la turbidité, et il a été étalonné par le SHOM avant d'être utilisé en mer lors d'une campagne sur le Pourquoi Pas dans le golfe de Gascogne.

L'étalonnage, effectué au SHOM, a permis d'évaluer la correction à apporter à la position du laser mesurée sur le PSD en fonction de la température, la pression

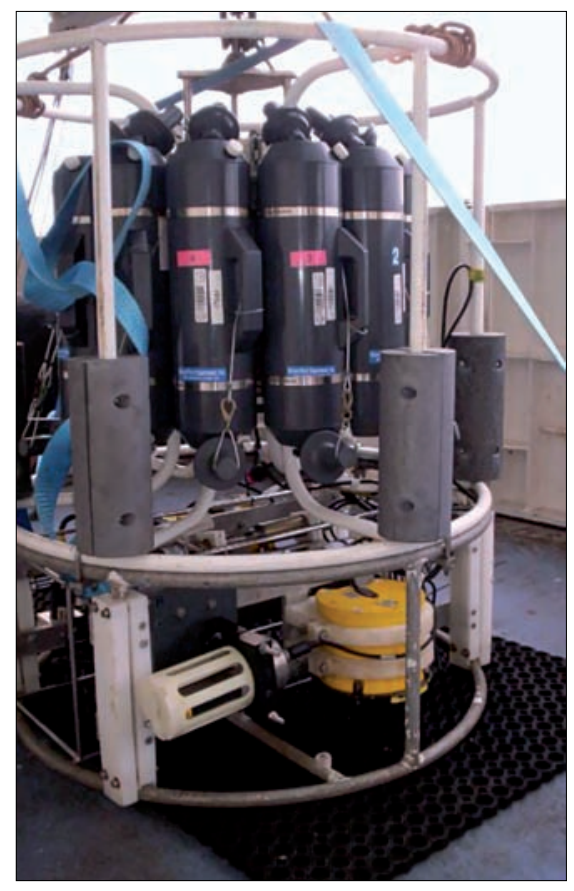

et la longueur d'onde. Le résultat de l'étalonnage a montré une relation quasilinéaire entre l'indice de réfraction et la correction apportée sur la position du PSD.

\section{Une première mondiale}

\section{lors de la campagne PROTEVS}

Le prototype étant prêt pour des essais in situ, ceux-ci ont été réalisés au printemps 2010, par le SHOM, lors de la campagne océanographique PROTEVS-2010 à bord du Pourquoi Pas [3] (figure 3).

Le prototype est monté en position horizontale (comme sur la figure 3) ou verticale, sous un carrousel de bouteilles de prélèvement piloté de la surface, appelé «rosette ». Plusieurs profils de salinité ont été relevés: profils petits fonds dans les panaches de la Loire et de la Garonne,

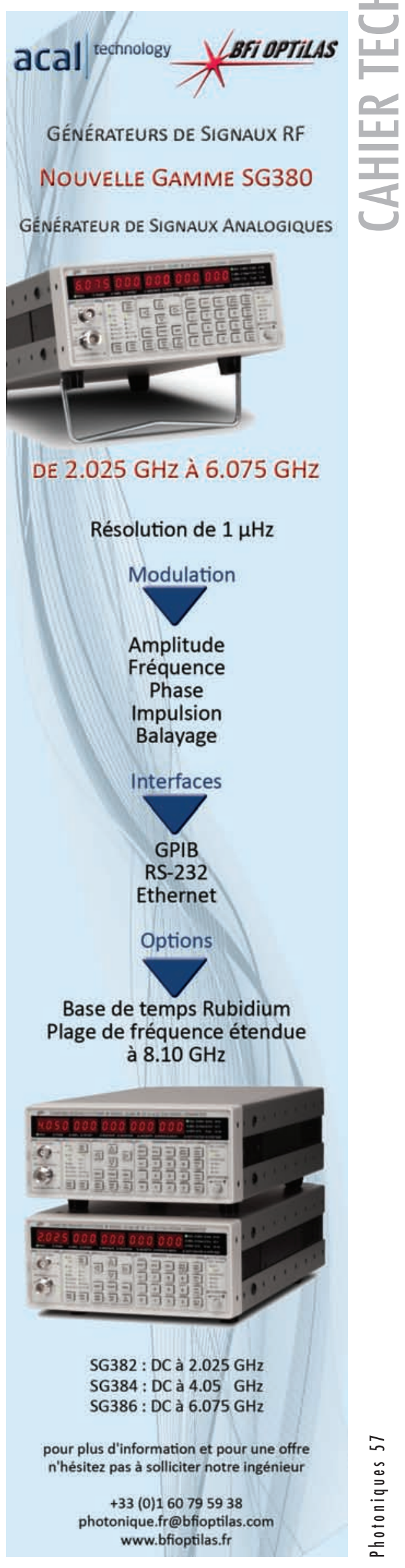




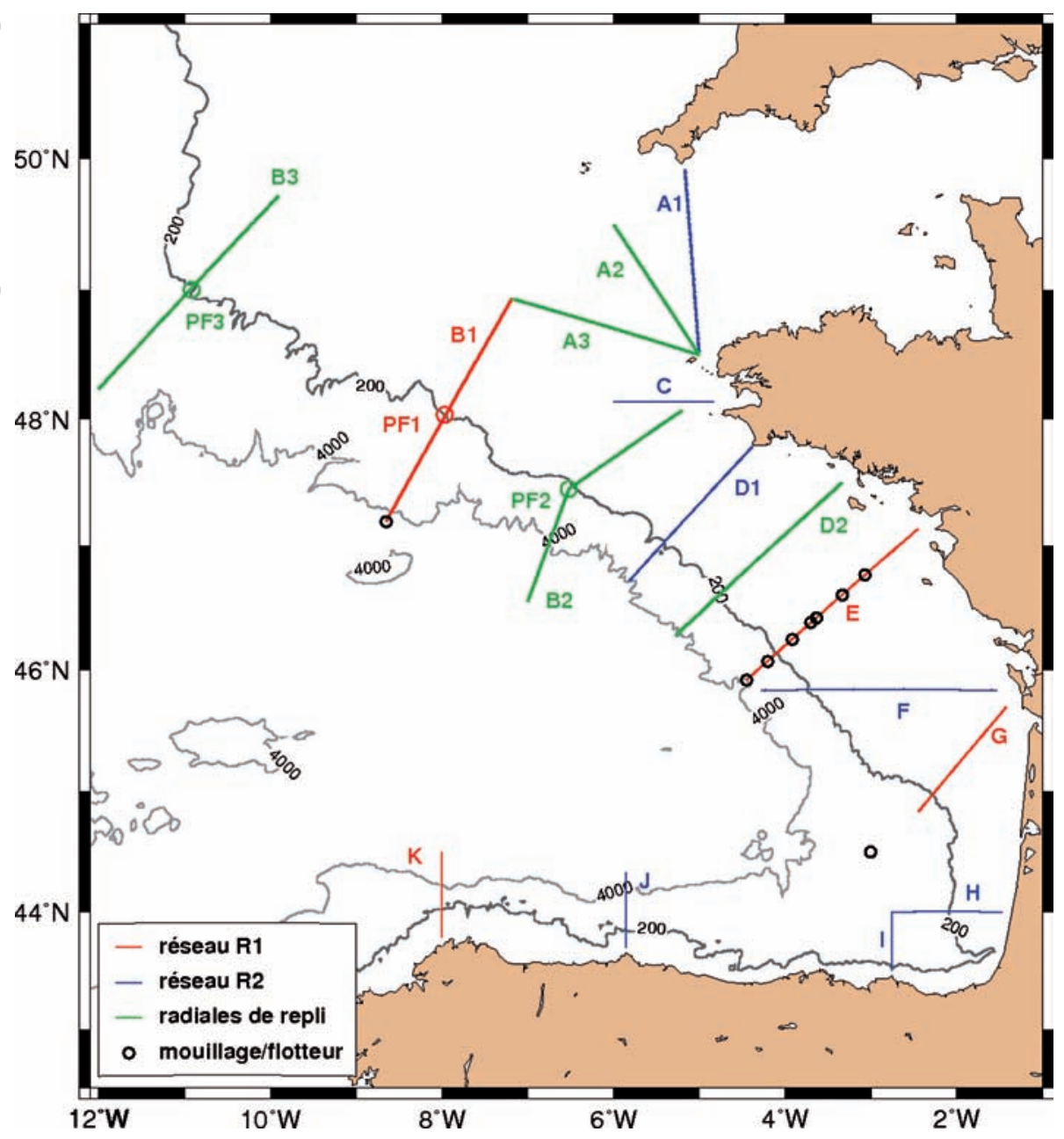

Figure 4. Campagne PROTEUS-2010 : cartographie des mesures réalisées dans le golfe de Gascogne.

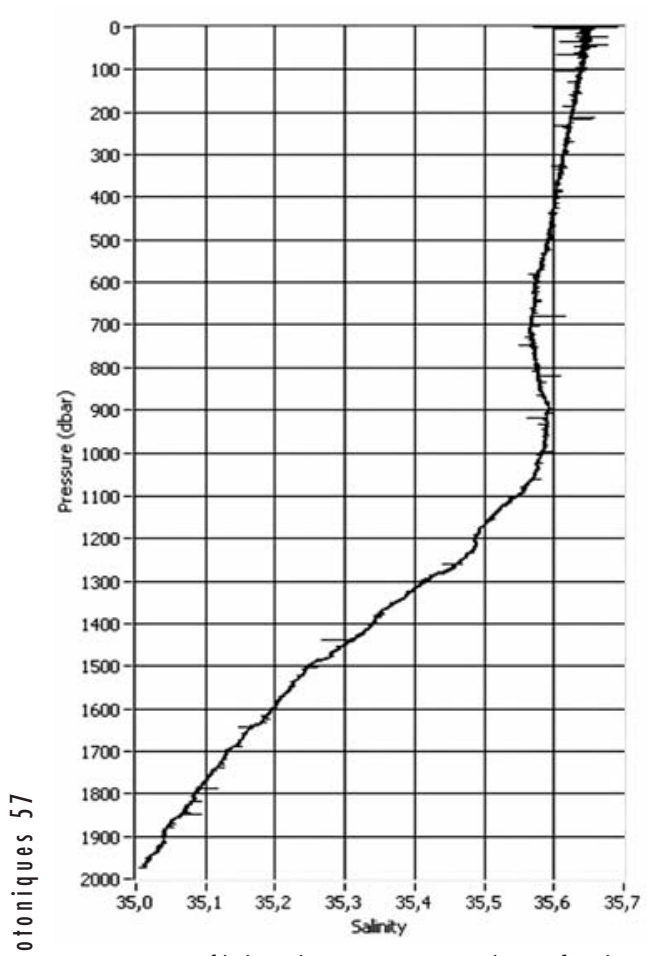

Figure 5. Profil de salinité à $2000 \mathrm{~m}$ de profondeur.

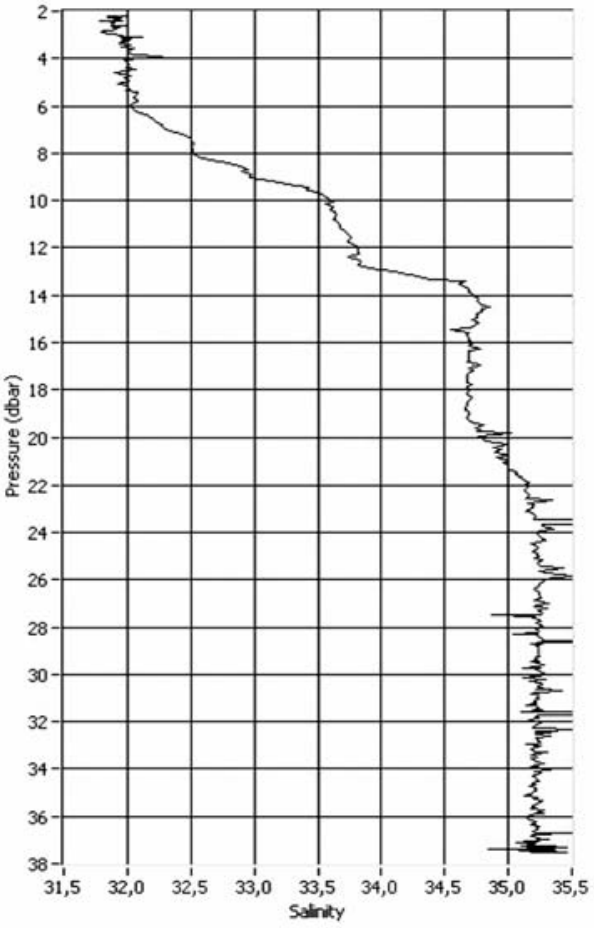

Figure 6. Profil côtier. et profils sur une profondeur de $2000 \mathrm{~m}$ dans le golfe de Gascogne (figure 4).

Un profil à $2000 \mathrm{~m}$ de profondeur, constituant une première mondiale, a été obtenu à une fréquence d'acquisition de $5 \mathrm{~Hz}$ et une vitesse de descente de $0,5 \mathrm{~m} / \mathrm{s}$, dans une zone où les variations de masse volumique sont principalement dues aux variations de température (figure 5). Ce profil a été converti en valeurs de salinité. La composition de l'eau de mer de la zone étant proche de celle d'une eau standard, il est similaire à celui obtenu par un profileur CTD de référence et les mêmes petites variations de salinité peuvent y être observées, démontrant une sensibilité de mesure proche de celle des capteurs de conductivité, réputée pourtant inaccessible par les technologies optiques

Un second profil côtier a été réalisé à la même vitesse de descente (figure 6). Dans ce cas, les variations de masse volumique sont davantage liées aux variations de salinité. De nouveau, le réfractomètre a démontré sa sensibilité aux petites variations de salinité, que l'on peut observer en référence sur le profil CTD. Ces résultats obtenus dans des conditions sévères et les articles qui ont été publiés ont suscité un très vif regain d'intérêt pour les technologies optiques, en témoignent les nombreuses solutions basées sur ce principe et citations de ce travail.

\section{Références}

[1] McDougall T.J., R. Feistel, F.J. Millero, D.R. Jackett, D.G. Wright, B.A. King, G.M. Marion, C.-T.A. Chen and P. Spitzer, 'The International Thermodynamic Equation of Seawater 2010 (TEOS-2010): Calculation and Use of Thermodynamic Properties', Global Ship-based Repeat Hydrography Manual, IOCCP Report $n^{\circ} 14$, ICPO Publication Series n 134, 2009.

[2] SCOR/IAPSO Working Group 127 on Thermodynamics and Equation of state of Seawater, 'Refractive index of seawater', Meeting 6-11 May 2007 , Italy.

[3] Le Menn M., J.L. de Bougrenet de la Tocnaye, P. Grosso, L. Delauney, C. Podeur, P. Brault and $O$. Guillerme, Advances in measuring ocean salinity with an optical sensor, Meas. Sci. Technol. 22, 115202 (2011). 